PROCEEDINGS OF THE AMERICAN MATHEMATICAL SOCIETY

Volume 126, Number 5, May 1998, Pages 1515-1525

S 0002-9939(98)04155-0

\title{
SOME RESULTS ON THE CONVEX HULL OF FINITELY MANY CONVEX SETS
}

\author{
ALBERT BORBÉLY
}

(Communicated by Christopher Croke)

\begin{abstract}
A better than quadratic estimate is given for the volume of the convex hull of $n$ points on Hadamard manifolds with pinched curvature. It was known previously that the volume is bounded by some polynomial in $n$. The estimate comes from the study of the convex hull of finitely many convex sets on Hadamard manifolds.
\end{abstract}

\section{IntRoduction}

On complete simply connected manifolds with sectional curvature $K<-1$ convex sets played an important role in the solution of the Dirichlet problem at infinity. Choi $[\mathrm{CH}]$ proved that for such a manifold $M$ with the usual compactification $\bar{M}=M \cup S_{\infty}(M)$, where $S_{\infty}(M)$ denotes the ideal boundary (for details see [EO]), the Dirichlet problem at infinity is solvable if convex sets separate ideal points. That is, for any two ideal points $P, Q \in S_{\infty}(M)$ there is a closed (in $\bar{M}$ ) convex neighborhood $V$ of $P$ not containing $Q$.

In case of pinched curvatures [AN] (or with a suitable growth condition on the curvature [BO1]) one can always construct convex sets whose intersection with the ideal boundary is non-trivial. It can be shown, statement $\left(C_{1}\right)$, that every closed subset of the ideal boundary is the intersection of some convex set and the ideal boundary (see [AN, Theorem 3.3], [BO1, Theorem 2.3]). Suitable reparametrizations of the distance functions to these convex sets turn out to be subharmonic, and they will serve as barrier functions for the Perron method, which can be easily adapted to the situation at hand.

However, once we remove the lower bound on the curvature the situation changes dramatically and the above statement ([AN, Theorem 3.3], [BO1, Theorem 2.3]) is no longer true. It was shown recently that there are complete simply connected manifolds with sectional curvature $K<-1$ which possess a point on the ideal boundary with the property that the convex hull of every neighborhood is the whole manifold (see $[\mathrm{ANC}],[\mathrm{BO} 2]$ ); moreover there are manifolds such that every point on the ideal boundary has this property [ANC]. Clearly, for these manifolds convex sets cannot separate ideal points, in fact, it was shown that for these manifolds the Dirichlet problem at infinity is not solvable.

Received by the editors February 27, 1996 and, in revised form, October 14, 1996.

1991 Mathematics Subject Classification. Primary 53C20.

Key words and phrases. Convex hull.

This was research supported by the Kuwait University Research Grant SM 146.

(C)1998 American Mathematical Society 
Our first lemma (Lemma 1) can be considered as a possible generalization of the statement above $\left(C_{1}\right)$ to Hadamard manifolds with sectional curvature bounded away from 0 . Let us call a closed set in the ideal boundary convex if it is the intersection of some convex set of the manifold and the ideal boundary. Then Lemma 1 states that on the ideal boundary the finite union of convex sets is a convex set in this sense. The precise statement is the following.

Lemma 1. Let $M^{m}$ be a complete simply connected Riemannian manifold with sectional curvatures $K<-1$. Let $F_{1}, \ldots, F_{n}$ be convex sets, $F=\bigcup_{i=1}^{n} F_{i}$, and denote by $\operatorname{Chull}(F)$ the convex hull of $F$. Then there is a constant $C$ depending on the sets $F_{1}, \ldots, F_{n}$, such that for every $P \in \operatorname{Chull}(F)$, dist $(P, F)<C$.

For a Hadamard manifold we define the convex hull of a closed set to be the smallest closed convex set containing it in $\bar{M}=M \cup S_{\infty}(M)$.

This shows that, in general, the convex hull of finitely many points (on the ideal boundary) is "well behaved"; for example, it cannot be the whole manifold, which, in view of the examples $[\mathrm{ANC}]$ and [BO2], were not so obvious. Actually, Lemma 1 provides us with some estimate as to the "thickness" of the convex hull.

Lemma 1 can also be looked at as a new way to construct convex sets in a general Hadamard manifold with curvatures bounded away from 0 .

Using these ideas, in the pinched curvature case, we can estimate the volume of the convex hull of finitely many points. It was shown recently [BOW] that the volume of the convex hull of $n$ points is bounded by some polynomial in $n$ whose degree depends on the dimension and the pinching. We improve this by giving a better than quadratic estimate.

Theorem 1. Let $M=M^{m}$ be a complete simply connected Riemannian manifold with sectional curvatures $-k^{2}<K<-1, P_{i} \in M \cup S_{\infty}(M), i=1, \ldots, n$, and $F=\operatorname{Chull}\left\{P_{1}, \ldots, P_{n}\right\}$ the convex hull. Then we have the following estimate for the volume:

$$
\operatorname{Vol}(F) \leq C n^{2-\delta},
$$

where $\delta=\frac{1}{1+4 k(m-1)}$ and the constant $C=C(m, k)$ depends on the dimension and the pinching.

As a matter of fact, a simple observation (Lemma 2) combined with the ideas presented in [BOW] immediately yields an $O\left(n^{2} \log n\right)$ bound on the volume (see the argument at the beginning of Section 2).

It was speculated in [BOW] that it might be possible to give a linear bound (in $n$ ) for the volume of the convex hull of $n$ points. Theorem 1 seems to support this idea.

Throughout the paper we use notations which are fairly standard. We write $B(Q, r)$ for the geodesic ball of radius $r$ around $Q$ and $N(F, r)$ for the $r$-neighborhood of the set $F$. We also use various constants. Their dependence on certain parameters is indicated in parentheses like $C(m, k), C_{1}(m, k, r), \ldots$, etc.

\section{Convex hulls of CONVEX Sets}

Throughout this section $M$ denotes a Hadamard manifold with sectional curvatures bounded away from 0 , say $K<-1$. 
Proof of Lemma 1. By an approximation lemma of Greene and Wu [GW, Prop. 2.2] we may assume that each $\partial F_{i}$ is smooth. Let $h_{i}: M \rightarrow \mathbb{R}^{+}$be the reparametrized distance function to $F_{i}$; that is, for $P \in M, h_{i}(P)=f\left(\varrho_{i}(P)\right)$, where $f(t)=1-e^{-t}$ and $\varrho_{i}(P)=\operatorname{dist}\left(P, F_{i}\right)$. Then for the Hessian and the differential of $h_{i}$ we have

$$
d h_{i}=f^{\prime} d \varrho_{i}, \quad D^{2} h_{i}=f^{\prime \prime} d \varrho_{i} \otimes d \varrho_{i}+f^{\prime} D^{2} \varrho_{i} .
$$

Denoting $h=h_{1}+\ldots+h_{n}$ we will show that for a sufficiently small $\epsilon>0$ the set $\hat{F}=\{P \in M: h(P) \leq n-\epsilon\}$ is convex. This implies the theorem because Chull $(F) \subset \hat{F}$ and for every $P \in M, h(P)=n-\epsilon$, we have the following:

$$
\ln \frac{1}{\epsilon} \leq \operatorname{dist}(P, F) \leq \ln \frac{n}{\epsilon} \text {. }
$$

The constant $C$ in the statement of Lemma 1 can be choosen to be $C=\ln \frac{n}{\epsilon}$.

We prove the convexity of $\hat{F}$ by showing that $\partial \hat{F}$, which is the level set $h=n-\epsilon$, has positive definite 2 nd fundamental form.

Let $\alpha>0$ be a fixed "small" angle such that

$$
\sin ^{2} \alpha<1 / 2 \quad \text { and } \quad \cos ^{2} \alpha>3 / 4 \text {. }
$$

Then, due to the negativity of the sectional curvatures, from a large enough distance $C_{1}$ (which depends only on the upper bound of the curvature, $K \leq-1$ ) the viewing angle of every convex set will be less than $\alpha / 3$, as measured by the maximal angle subtended by two points in the set. Selecting a point $P_{i} \in F_{i}$ from each convex set we can find a distance $C_{2}$ such that the viewing angle of the set $\left\{P_{1}, \ldots, P_{n}\right\}$ is smaller than $\alpha / 3$ from this distance. Combining these together we have that from the distance $\max \left\{C_{1}, C_{2}\right\}$ the viewing angle of $F$ is less than $\alpha$.

This means, in view of (1.2), that there is an $\epsilon>0$ such that for every point $P \in M$ for which $h(P)=n-\epsilon$ we have $\measuredangle\left(\nabla h_{i}(P), \nabla h_{j}(P)\right)<\alpha$ which implies that

$$
\measuredangle\left(\nabla h_{i}(P), \nabla h(P)\right)<\alpha .
$$

Here the symbol $\measuredangle\left(\nabla h_{i}(P), \nabla h_{j}(P)\right)$ stands for the angle of the gradient vectors $\nabla h_{i}(P), \nabla h_{j}(P)$.

Let $X \in T_{P} M$ now be a unit vector tangent to the level set $h=n-\epsilon$; that is, $d h(X)=0$. From the inequality above it follows that $X$ is almost tangent to the level sets of $\varrho_{i}$ passing through $P$; that is

$$
d \varrho_{i}(X)<\sin \alpha .
$$

On the other hand, standard arguments, involving Jacobi fields, show that the level sets of $\varrho_{i}$ have a definite convexity; that is, denoting by $A_{i}$ the 2 nd fundamental form of the level set $\varrho_{i}=r$ we have $A_{i}>\tanh (r) I d$, where $I d$ denotes the identity matrix. Let $C_{3}$ be a constant, such that for $r>C_{3}$ we have $\tanh (r)>2 / 3$ and set $C_{4}=\max \left\{C_{1}, C_{2}, C_{3}\right\}$. Then for a small enough $\epsilon$, such that $\ln (1 / \epsilon)=C_{4}$ (or equivalently if $r=\operatorname{dist}(P, F) \geq C_{4}$, cf. (1.2)), in view of (1.3) and (1.4), we have

$$
D^{2} \varrho_{i}(X, X)>\tanh (r) \cos ^{2} \alpha>1 / 2, \quad i=1,2, \ldots, n .
$$

This, together with (1.1), (1.3) and (1.4), implies that $D^{2} h_{i}(X, X)>0$ for $i=1, \ldots, n$; therefore $D^{2} h(X, X)>0$, which concludes the proof of the theorem.

Remark. If $\bigcap_{i=1}^{n} F_{i} \neq \emptyset$, then it is possible to make the dependence of the constant $C$ in Lemma 1 on the sets $F_{1}, \ldots, F_{n}$ more precise. From (1.2) and the definition of $\epsilon$ it is clear that $C=C_{4}+\ln n$. On the other hand $C_{4}$ depends only on $C_{1}, C_{2}$ and $C_{3}$. Out of these $C_{3}$ is an absolute constant and $C_{1}$ depends only on the upper 
bound of the sectional curvatures. In case $\bigcap_{i=1}^{n} F_{i} \neq \emptyset$ let $P \in \bigcap_{i=1}^{n} F_{i}$ be any point and set $P_{i}=P$ for $i=1, \ldots, n$. Then we can choose $C_{2}=0$. This means that if $\bigcap_{i=1}^{n} F_{i} \neq \emptyset$, then for every $P \in \operatorname{Chull}(F)$, $\operatorname{dist}(P, F)<C_{4}+\ln n$, where the constant $C_{4}$ depends only on the upper bound of the curvature.

The examples in $[\mathrm{ANC}]$ and $[\mathrm{BO} 2]$ show that the $\ln n$ term cannot be removed altogether.

In the presence of a lower bound on the curvature the following stronger version of Lemma 1 is known.

Lemma 2. Let $M=M^{m}$ be a complete simply connected Riemannian manifold with sectional curvatures $-k^{2}<K<-1, F_{1}, \ldots, F_{n}$ be convex sets with $\bigcap_{i=1}^{n} F_{i} \neq \emptyset$ and set $F=\bigcup_{i=1}^{n} F_{i}$. Then there is a constant $C=C(k)$ depending only on the pinching, such that the convex hull of $F$ lies in the $C$-neighborhood of $F$.

Proof. It is enough to observe that $F$ is quasiconvex; that is, any geodesic connecting two points in $F$ remains within a fixed distance (independent of $k$ ) of $F$. In [BOW1] (for a brief explanation see also [BOW, p. 65]) it was shown that in such a case $\operatorname{Chull}(F)$ lies in a uniform $C$-neighborhood of $F$, where $C$ depends only on $k$. This concludes the proof of Lemma 2 .

\section{Volume of CONVEX Hulls}

Throughout this section we assume that the manifold $M$ has pinched negative curvature.

Before we start the proof of Theorem 1 let us show how ideas from [BOW] combined with Lemma 2 give us an almost quadratic estimate for the volume of $F$, the convex hull of $n$ points $P_{1}, \ldots, P_{n}$. Let $R \in F$ be any point. Then $F$ can be considered as the convex hull of the geodesic segments $\overline{R P_{i}}=F_{i}, i=1, \ldots, n$. Then Lemma 2 shows that $F$ lies inside a $C$-neighborhood of the geodesic segments $\overline{R P_{i}}$, $i=1, \ldots, n$, where the constant $C$ depends only on the pinching. In other words, the convex hull of $n$ points always lies inside some $n$ uniform tubes centered around rays connecting an arbitrary point inside the convex hull to the given points. Moreover, the radius of these tubes depends only on the pinching constant.

In the presence of a lower bound on the curvature the volume of finite pieces of these tubes can be estimated readily, which gives us the following bound on the volume of $F \cap B_{r}$, where $B_{r}$ denotes some ball of radius $r$ :

$$
\operatorname{Vol}\left(F \cap B_{r}\right)<\tilde{C} n r .
$$

The constant $\tilde{C}$ depends on the pinching and the dimension of the manifold only.

The proof of the volume estimate (Th. 4.1) in $[\mathrm{BOW}]$ is based on the observation (Lemma 4.5) that the convex hull $F$ lies inside some tubes and uniform balls. The volume of these tubes are bounded, the balls have radii $O(\log n)$ and the total number of tubes and balls is less than $3 n$. Combining this with the volume estimate above we immediately have an almost quadratic estimate for the volume of the convex hull of $n$ points,

$$
\operatorname{Vol}(F)=O\left(n^{2} \log n\right) .
$$

Although Theorem 1 does not improve this estimate significantly, the method of proof is completely different from that of [BOW], and this may turn out to be useful in getting the linear volume estimate. 
In the proof of Theorem 1 we have to estimate the volume of a layer, with a given thickness, over some convex set. To this end, we need the following observation.

Proposition 1. Let $M=M^{m}$ be a complete simply connected manifold with sectional curvatures $-k^{2}<K<-1, B_{r}$ be a closed geodesic ball of radius $r>0$ and $G \subset B_{r}$ be a closed convex set. Then there is a constant $C=C(m, k, r)$, depending on the dimension of $M$, the pinching and on the radius of the ball, such that

$$
\operatorname{Vol}(N(G, \delta)-G)<\delta C,
$$

where $N(G, \delta)$ denotes the $\delta$-neighborhood of $G$ in $B_{r}$.

Proof of Proposition 1. Denote by $S_{r}=\partial B_{r}$ the boundary sphere of $B_{r}$, and for any closed convex set $A \subset B_{r}$ let $\pi: S_{r} \rightarrow \partial A$ be the nearest point retraction to $\partial A$. The negativity of the curvature implies that $\pi: S_{r} \rightarrow \partial A$ is a distance decreasing (and onto) map. As an immediate consequence of this, we have

$$
\operatorname{Hf}_{i}(\partial A) \leq \operatorname{Hf}_{i}\left(S_{r}\right), \quad i \geq 1
$$

where $\operatorname{Hf}_{i}($.$) denotes the i$-th dimensional Hausdorff measure.

Assume now that $\partial G$ is a smooth hypersurface. Then combining the coarea formula and the inequality above we have

$$
\operatorname{Vol}(N(G, \delta)-G)=\int_{N(G, \delta)-G}|d \varrho| d v_{g}=\int_{0}^{\delta} \operatorname{Vol}_{m-1}\left(\varrho^{-1}(t)\right) d t \leq \delta \operatorname{Vol}_{m-1}\left(S_{r}\right),
$$

where $v_{g}$ is the volume form on $M$ and $\varrho$ is the distance function to $G$. This proves the proposition in this case.

If $G$ has no interior points, then it must be totally geodesic and as $\operatorname{such}_{n} \operatorname{Hf}_{n}(G)=$ 0 . Therefore we can apply the above argument to a sufficiently small convex tubular neighborhood of $G$ with a smooth boundary.

In case $G$ has nonempty interior we have seen already that $\operatorname{Hf}_{n}(\partial G) \leq \operatorname{Hf}_{n}\left(S_{r}\right)=$ 0. Then from the approximation theorem of Greene and Wu [GW, Prop. 2.2] (applied to the distance function to $G$ ) one can approximate $G$ sufficiently closely by a convex set $\tilde{G} \supset G$ with smooth boundary. The fact that $\operatorname{Hf}_{n}(\partial G)=0$ implies that $\operatorname{Vol}(\tilde{G}-G)$ can be as small as we want. Since

$$
\operatorname{Vol}(N(G, \delta)-G)<\operatorname{Vol}(N(\tilde{G}, \delta)-\tilde{G})+\operatorname{Vol}(\tilde{G}-G)
$$

the proposition is proved by applying the previous argument to $\tilde{G}$.

Since the conjecture may very well be true without the lower curvature bound it is important to see where it is used. The proof of Theorem 1 consists of two parts. In the first part a convex set is constructed (by a method similar to that of Lemma 1) which is used to approximate the convex hull. For this we do not need the lower curvature bound. It comes into play, in an essential way, only through the various volume estimates of the second part. As far as the geometry of the convex hull is concerned we could, probably, do very well without the lower curvature bound, but we do not see how to circumvent it when it comes to volume estimates.

Proof of Theorem 1. We proceed by induction. The key step is to estimate how much larger the volume of the convex hull gets by adding one more point. Without loss of generality we may assume that $P_{1}, \ldots, P_{n} \in S_{\infty}(M)$ and set $F=$ $\operatorname{Chull}\left\{P_{1}, \ldots, P_{n-1}\right\}$ and $F_{1}=\operatorname{Chull}\left(F, P_{n}\right)$. 
Let $Q \in \partial F$ be the "closest point" to $P_{n}$, that is, the point where the Busemann function at $P_{n}$ assumes its minimum on $\partial F$, and denote by $\gamma=\{\gamma(t): t \in[0, \infty)\}$ the unit speed geodesic ray connecting $Q$ to $P_{n}$. Then clearly $\gamma$ is perpendicular to $\partial F$. In case $\partial F$ is not smooth by this we mean that $\measuredangle(S Q T) \geq \pi / 2$ for every $S \in \gamma, T \in F$.

Using a similar method to that of Lemma 1 we are going to construct a convex set $H \supset F_{1}$ whose volume can be estimated. As in the previous section $H$ will be the sublevel set of some function constructed from reparametrized distance functions. However, this time, the function we use to reparametrize the distance function will include a constant $a$, which, for the purposes of this proof, can be taken to be $a=1 / 4$. Nevertheless, with the possibility of further applications in mind, we prefer to use $a$ instead of $1 / 4$.

More precisely, let $h_{1}, h_{2}: M \rightarrow \mathbb{R}^{+}$be reparametrized distance functions to $F$ and $\gamma$, respectively; that is, for $P \in M, h_{i}(P)=f\left(\varrho_{i}(P)\right), i=1,2$, where $f(t)=1-e^{-a t}, a=1 / 4, \varrho_{1}(P)=\operatorname{dist}(P, F)$ and $\varrho_{2}(P)=\operatorname{dist}(P, \gamma)$. Set $h=h_{1}+h_{2}$ and let $H=\{P \in M: h(P) \leq 1\}$ be a sublevel set of $h$.

Because $\partial H$ is not necessarily smooth we cannot show the convexity of $H$ by arguing that the 2 nd fundamental form of its boundary is positive definite. However, we can find a smooth "enveloping" surface with a positive 2 nd fundamental form. More precisely, for every point $P \in \partial H$, we will construct a set $\tilde{H} \supset H$ such that $P \in \partial \tilde{H}, \partial \tilde{H}$ is smooth near $P$ and $\partial \tilde{H}$ has a positive 2 nd fundamental form at $P$. This will imply the convexity of $H$.

So, let $P \in \partial H,(h(P)=1)$ and denote by $S$ and $T$ the closest points to $P$ on $\gamma$ and $F$, respectively, and let $s=\operatorname{dist}(P, S)$ and $t=\operatorname{dist}(P, T)$; see Figure 1 . First we replace $h_{1}$ with a smooth function $\tilde{h}_{1} \leq h_{1}$ in the following way. Let $V_{1}$ be the closed half-space at $T$ containing $F$, namely $V_{1}$ is the collection of geodesic rays emanating from $T$ and having angle larger than or equal to $\pi / 2$ with the geodesic segment $T P$. Denote by $\tilde{\varrho}_{1}$ the distance function from $V_{1}$ and define $\tilde{h}_{1}$ by $\tilde{h}_{1}=f\left(\tilde{\varrho}_{1}\right)$. Now, clearly $\partial V_{1}$ is smooth and so are the functions $\tilde{\varrho}_{1}$ and $\tilde{h}_{1}$. Moreover, from $V_{1} \supset F$ we have $\tilde{h}_{1} \leq h_{1}$.

Next we replace $h_{2}$ with a function $\tilde{h}_{2}$ which is smooth near $P$ and $\tilde{h}_{2} \leq h_{2}$. Similarly, let $V_{2}$ be the closed half space at $Q$ perpendicular to $\gamma$ and containing the set $F$. If $P \notin \partial V_{2}$, then $h_{2}$ is smooth near $P$ and we set $\tilde{h}_{2}=h_{2}$. If $P \in \partial V_{2}$, then let $\tilde{\gamma}$ be the extension of the geodesic ray $\gamma$ beyond $Q$; that is, for some small $\epsilon>0, \tilde{\gamma}=\gamma[-\epsilon, \infty]$ and set $\tilde{h}_{2}=f\left(\tilde{\varrho}_{2}\right)$, where $\tilde{\varrho}_{2}$ denotes the distance function from $\tilde{\gamma}$. Again $\tilde{\gamma} \supset \gamma$ implies $\tilde{h}_{2} \leq h_{2}$.

Let $\tilde{h}=\tilde{h}_{1}+\tilde{h}_{2}$ and $\tilde{H}=\{R \in M: \tilde{h}(R) \leq 1\}$. Clearly $\tilde{h}(P)=h(P)$ and $\tilde{h} \leq h$, which implies that $\tilde{H} \supset H$ and $P \in \partial \tilde{H}, \partial H$. We now have to show that $\partial \tilde{H}$ has positive definite 2 nd fundamental form at $P$ or, equivalently, that the Hessian of $\tilde{h}$, $D^{2} \tilde{h}$, is positive definite on vectors tangent to $\partial \tilde{H}$.

From the construction it follows that $\measuredangle(P T Q) \geq \pi / 2$ and $\measuredangle(P S Q)=\pi / 2$ (if $S \neq Q)$; therefore, on account of the negative curvature, we have $\measuredangle(S P T) \leq \pi / 2$. Setting $N_{i}=\nabla \tilde{\varrho}_{i}(P), i=1,2$, this means that

$$
\left\langle N_{1}, N_{2}\right\rangle>0, \quad \text { and } \quad\left\|N_{i}\right\|=1, i=1,2,
$$

where $\langle\cdot, \cdot\rangle$ denotes the Riemannian metric; see Figure 1. 


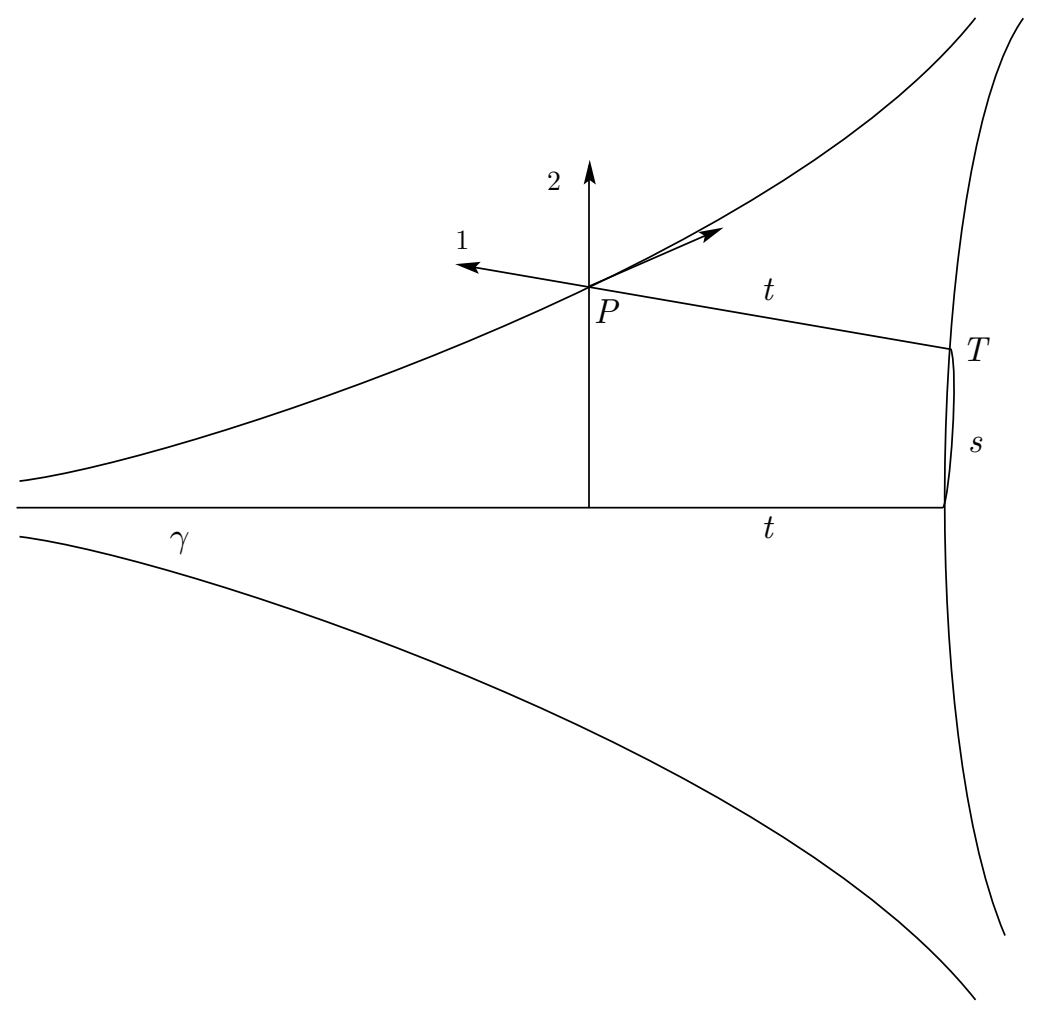

FiguRE 1

Let $X$ be a unit tangent vector tangent to $\partial \tilde{H}$ at $P$, that is, $\langle\nabla \tilde{h}, X\rangle=0$; then using (1.1) and the fact that $h(P)=1$ we have

(2.2) $\left\langle e^{-a t} N_{1}+e^{-a s} N_{2}, X\right\rangle=0, \quad$ where $e^{-a t}+e^{-a s}=1 \quad$ and $\quad\|X\|=1$.

From (2.1) one gets

$$
\left\|e^{-a s} N_{1}-e^{-a t} N_{2}\right\| \leq \sqrt{\left(e^{-a s}\right)^{2}+\left(e^{-a t}\right)^{2}} .
$$

Since $\left\langle N_{1}, X\right\rangle$ and $\left\langle N_{2}, X\right\rangle$ have opposite signs (cf. (2.2)) and $\|X\|=1$ we have

$$
\sqrt{\left(e^{-a s}\right)^{2}+\left(e^{-a t}\right)^{2}} \geq\left|\left\langle e^{-a s} N_{1}-e^{-a t} N_{2}, X\right\rangle\right|=e^{-a s}\left|\left\langle N_{1}, X\right\rangle\right|+e^{-a t}\left|\left\langle N_{2}, X\right\rangle\right| .
$$

Using (2.2) again implies that

$$
\left(e^{-a s}+\frac{\left(e^{-a t}\right)^{2}}{e^{-a s}}\right)\left|\left\langle N_{1}, X\right\rangle\right| \leq \sqrt{\left(e^{-a s}\right)^{2}+\left(e^{-a t}\right)^{2}}
$$

and

$$
\left(e^{-a t}+\frac{\left(e^{-a s}\right)^{2}}{e^{-a t}}\right)\left|\left\langle N_{2}, X\right\rangle\right| \leq \sqrt{\left(e^{-a s}\right)^{2}+\left(e^{-a t}\right)^{2}},
$$

which yields

$$
\left|\left\langle N_{1}, X\right\rangle\right| \leq \frac{e^{-a s}}{\sqrt{\left(e^{-a s}\right)^{2}+\left(e^{-a t}\right)^{2}}}, \quad\left|\left\langle N_{2}, X\right\rangle\right| \leq \frac{e^{-a t}}{\sqrt{\left(e^{-a s}\right)^{2}+\left(e^{-a t}\right)^{2}}} .
$$


Taking into account that $e^{-a t}+e^{-a s}=1$ we have

$$
\left|\left\langle N_{1}, X\right\rangle\right| \leq \sqrt{2} e^{-a s}, \quad\left|\left\langle N_{2}, X\right\rangle\right| \leq \sqrt{2} e^{-a t} .
$$

We now estimate the Hessian of $\tilde{h}$ at $P$ in the direction of $X$. From (1.1) we have

$$
\begin{aligned}
D^{2} \tilde{h}(X, X)= & f^{\prime \prime}(t)\left\langle N_{1}, X\right\rangle^{2}+f^{\prime}(t) D^{2} \tilde{\varrho}_{1}(X, X)+f^{\prime \prime}(s)\left\langle N_{2}, X\right\rangle^{2} \\
& +f^{\prime}(s) D^{2} \varrho_{2}(X, X) .
\end{aligned}
$$

Because of the upper bound of the curvature one can estimate $D^{2} \varrho_{i}(X, X)$ using standard comparison technique as follows:

$$
D^{2} \tilde{\varrho}_{i}(X, X) \geq\left(1-\left\langle N_{i}, X\right\rangle^{2}\right) \tanh \left(\varrho_{i}(P)\right), \quad i=1,2 .
$$

This implies

$$
\begin{aligned}
D^{2} \tilde{h}(X, X) & \geq-a^{2}\left(e^{-a t}\left\langle N_{1}, X\right\rangle^{2}+e^{-a s}\left\langle N_{2}, X\right\rangle^{2}\right) \\
& +a e^{-a t}\left(1-\left\langle N_{1}, X\right\rangle^{2}\right) \tanh (t)+a e^{-a s}\left(1-\left\langle N_{2}, X\right\rangle^{2}\right) \tanh (s) .
\end{aligned}
$$

To prove that the right hand side of (2.4) is positive it suffices to show that

$$
\begin{aligned}
a^{2}\left(e^{-a t}\left\langle N_{1}, X\right\rangle^{2}+e^{-a s}\left\langle N_{2}, X\right\rangle^{2}\right) & <a e^{-a t}\left(1-\left\langle N_{1}, X\right\rangle^{2}\right) \tanh (t) \\
& +a e^{-a s}\left(1-\left\langle N_{2}, X\right\rangle^{2}\right) \tanh (s) .
\end{aligned}
$$

Therefore, by (2.3), it is enough to have

$$
2 a^{2} e^{-a t} e^{-a s}<a e^{-a t}\left(1-\left\langle N_{1}, X\right\rangle^{2}\right) \tanh (t)+a e^{-a s}\left(1-\left\langle N_{2}, X\right\rangle^{2}\right) \tanh (s) .
$$

The expression is symmetric in $s$ and $t$ and $e^{-a t}+e^{-a s}=1$ (see (2.2)), therefore we might as well assume that $e^{-a t} \leq 1 / 2$. Since $N_{i}$ and $X$ are unit vectors, both terms on the right hand side of the above inequality are non-negative so it is enough to have

$$
2 a^{2} e^{-a t} e^{-a s}<a e^{-a s}\left(1-\left\langle N_{2}, X\right\rangle^{2}\right) \tanh (s) .
$$

In view of (2.3) and $e^{-a t} \leq 1 / 2$ this follows from

$$
2 a^{2} e^{-a t} e^{-a s}<\frac{a}{2} e^{-a s} \tanh (s)
$$

that is

$$
4 a e^{-a t}<\tanh (s)
$$

A simple calculation shows that either $\tanh (s)>s / 2$ or $\tanh (s)>1 / 2$. In the first case $(\tanh (s)>s / 2)$ we have the following estimate: $e^{-a t}=1-e^{-a s}<a s$, which reduces $(2.5)$ to

$$
4 a^{2} s<\frac{s}{2}
$$

This is true for $a=1 / 4$.

In the second case $(\tanh (s)>1 / 2)(2.5)$ simply reduces to

$$
4 a e^{-a t}<\frac{1}{2}
$$

Taking into account that $e^{-a t} \leq 1 / 2$ and $a=1 / 4$ this will also be true. Combining these together means that the inequality (2.5) is satisfied, which in turn proves the convexity of $\partial \tilde{H}$ at $P$ and therefore the convexity of the set $H$. 
This concludes the first part of the proof. Until now we have not used the lower curvature bound. However, it will be used in the second part every time we need to estimate volume. Apart from the volume estimates it is used only once in an argument needing Lemma 2 . Since the argument ends with a volume estimate there is no point in trying to replace Lemma 2 with Lemma 1.

To estimate $\operatorname{Vol}(H)$ we divide $H$ into three parts. By an elementary computation, involving triangle comparisons with the Euclidean space, one can easily see that there is an absolute constant $r_{0}>0$, such that, for every $r>r_{0}$, the ball $B(Q, r)$ of radius $r$ around $Q$ disconnects the set $H$ in such a way that no point of $\gamma-B(Q, r)$ can be connected to any point of $F-B(Q, r)$ by a curve lying entirely in the set $H-B(Q, r)$. We denote by $H_{1}$ the connected component of $H-B(Q, r)$ containing $\gamma-B(Q, r)$, by $H_{3}$ the rest of $H-B(Q, r)$ (not necessarily connected) and by $H_{2}$ the part of $H$ which lies inside the ball $B(Q, r) ; H_{2}=H \cap B(Q, r)$.

First we estimate the volume of $H_{1}$ (the long thin tube around $\gamma[r, \infty]$ ). For $P \in$ $\partial H_{1}-B(Q, r)$ let $S, T$ be, as usual, the closest points to $P$ on $\gamma$ and $F$, respectively, and write $s=\operatorname{dist}(P, S), s^{\prime}=\operatorname{dist}(Q, T), t=\operatorname{dist}(P, T)$ and $t^{\prime}=\operatorname{dist}(S, Q)$; see Figure 1. On account of the negative curvature we have $s^{\prime}<s, t^{\prime}<t$. From the construction of $H_{1}$ one can see that $s<t, r \leq \operatorname{dist}(P, Q) \leq t+s^{\prime}<t+s$ and $e^{-a s}+e^{-a t}=1$ (which follows from $h(P)=1$ ). Then an elementary computation shows that for a large enough $r_{0}$, we have $s<1 / 2$, which implies $t>t^{\prime}>t-1$ and $t^{\prime}>r-3 / 2$. Moreover, we have

$$
e^{-a t}=1-e^{-a s}>e^{-a} a s,
$$

which yields

$$
s<\frac{e^{-a(t-1)}}{a}<\frac{e^{-a\left(t^{\prime}-1\right)}}{a} .
$$

By a volume comparison theorem with initial submanifold $\gamma$ (due to E. Heintze and H. Karcher [HK] (see also Th. 7.5 in [CHA])) one can estimate the volume of the tube $H_{1}$, whose radius $s$ is bounded by (2.6), as follows:

$$
\operatorname{Vol}\left(H_{1}\right)<\tilde{C}(m, k) \int_{r-2}^{\infty} \frac{e^{-a\left(t^{\prime}-1\right)}}{a} d t^{\prime}<C(m, k) e^{-a r},
$$

where $C(m, k)$ and $\tilde{C}(m, k)$ denote appropriate constants depending on the dimension and the pinching.

Next we estimate the volume of $H_{3}$ by estimating the volume of $H_{3}-F$. Let $P, S, T$ and $s, s^{\prime}, t, t^{\prime}$ be the same as above. In this case, however, we have $t<s$ which, by a simillar argument as above, yields

$$
t<\frac{e^{-a(s-1)}}{a}<\frac{e^{-a\left(s^{\prime}-1\right)}}{a} \text { and } s^{\prime}>r-\frac{3}{2} .
$$

That is, $H_{3}-F$ is a "thin" layer over $F$ whose thickness approaches 0 exponentially as $s^{\prime}$ (the distance from $Q$ ) approaches infinity. To estimate the volume of this layer first we cut it into bounded pieces. As a consequence of Lemma 2 there is a constant $C=C(k)$ such that $F$ lies in the tubular neighborhood of the rays $\overline{Q P_{i}}, i=1, \ldots, n-1$, with radius $C$. On the other hand from the construction of $H$ one can see easily that $H$ remains within a distance $C_{1}=4 \ln 2$ of the set $\gamma \cup F$. Let us denote by $Q_{i}(x), i=1, \ldots, n-1$, the point on the geodesic ray $\overline{Q P_{i}}$ with $\operatorname{dist}\left(Q, Q_{i}(x)\right)=x$. Then, if $r_{0}$ is larger than a sufficiently large absolute constant 
and assuming that $C>C_{1}$, the set $F-B(Q, r)$, as well as the set $H_{3}$, will be covered by the collection of balls $B\left(Q_{i}\left(x_{l}\right), 2 C\right), i=1, \ldots, n-1$, and $x_{l}=r+l(C / 2)$, $l=1,2, \ldots$. For each of these balls we estimate $\operatorname{Vol}\left(\left(H_{3}-F\right) \cap B\left(Q_{i}\left(x_{l}\right), 2 C\right)\right)$.

Proposition 1 implies that for any ball $B_{q}$ of radius $q$ we have

$$
\operatorname{Vol}\left((N(F, \delta)-F) \cap B_{q}\right)<\delta C(m, k, q) .
$$

The thickness of the layer $H_{3}-F$ inside the ball $B\left(Q_{i}\left(x_{l}\right), 2 C\right)$ is bounded by $(2.8)$. Therefore we have

$$
\operatorname{Vol}\left(\left(H_{3}-F\right) \cap B\left(Q_{i}\left(x_{l}\right), 2 C\right)\right)<C(m, k) e^{-a x_{l}},
$$

which gives us for the total volume

$$
\operatorname{Vol}\left(H_{3}-F\right)<n \sum_{l} C(m, k) e^{-a x_{l}}<C_{1}(m, k) n e^{-a r} .
$$

Then for the volume of $H_{3}$ we have

$$
\operatorname{Vol}\left(H_{3}\right)<C_{1}(m, k) n e^{-a r}+\operatorname{Vol}(F) .
$$

At last, it remains to estimate the volume of $H_{2}=H \cap B(Q, r)$. We simply write

$$
\operatorname{Vol}\left(H_{2}\right)<\operatorname{Vol}\left(B(Q, r)<C_{2}(m, k) e^{k(m-1) r} .\right.
$$

Combining the estimates (2.7), (2.9) and (2.10), we have

$$
\begin{aligned}
\operatorname{Vol}\left(F_{1}\right)<\operatorname{Vol}(H) & <\operatorname{Vol}(F)+C(m, k) e^{-a r}+C_{1}(m, k) n e^{-a r}+ \\
& +C_{2}(m, k) e^{k(m-1) r},
\end{aligned}
$$

for any $r>r_{0}$.

Fixing $r$ would immediately give us $\operatorname{Vol}\left(F_{1}\right)<\operatorname{Vol}(F)+C_{4}(m, k) n$, which would imply $\operatorname{Vol}\left(F_{1}=\operatorname{Chull}\left(P_{1}, \ldots, P_{n}\right)\right)=O\left(n^{2}\right)$. We can do somewhat better by choosing

$$
r=\frac{\ln n}{k(m-1)+a} .
$$

With this choice an easy computation gives that

$$
\operatorname{Vol}\left(F_{1}\right)<\operatorname{Vol}(F)+C_{5}(m, k) n^{1-\frac{a}{a+k(m-1)}},
$$

which implies that

$$
\operatorname{Vol}\left(F_{1}\right)=O\left(n^{2-\frac{a}{a+k(m-1)}}\right) .
$$

This completes the proof of the theorem.

Remark. Using the more general reparametrized distance function of Theorem 1, in the proof of Lemma 1, one might improve it as follows.

Let $\hat{F}=\{P \in M: h(P) \leq n-1\}$. Then by an argument similar to the one given in (2.1) through (2.5), we believe it is possible to show that for a small enough $a$ (depending on the sets $\left.F_{i}, i=1, \ldots, n-1\right) \hat{F}$ will be convex. This implies not only that $\operatorname{Chull}(F)$ is in some uniform neighborhood of $F$, but it also shows that the "layer" Chull $(F)-F$ gets very "thin" at those points of $F_{i}$ which are far from the other sets. Although we did not state it formally, we used this property of the convex hull to prove Theorem 1. 


\section{REFERENCES}

[ANC] A. Ancona, Convexity at infinity and Brownian motion on manifolds with unbounded negative curvature, Rev. Mat. Iberoamericana 10 (1994), 189-220. MR 95a:58132

[AN] M.T. Anderson, The Dirichlet problem at infinity for manifolds of negative curvature, J. Differential Geometry 18 (1983), 701-721. MR 85m:58178

[BO1] A. Borbély, A note on the Dirichlet problem at infinity for manifolds of negative curvature, Proc. Amer. Math. Soc. 114 (1992), 865-872. MR 92f:58180

[BO2] A. Borbély, The nonsolvability of the Dirichlet Problem on negatively curved manifolds, Differ. Geom. and Appl., to appear.

[BOW] B.H. Bowditch, Some results on the geometry of convex hulls in manifolds of pinched negative curvature, Comment. Math. Helvetici 69 (1994), 49-81. MR 94m:53044

[BOW1] B.H. Bowditch, Geometrical finiteness with variable negative curvature, Preprint, I.H.E.S. (1990).

[CHA] I. Chavel, Riemannian Geometry - A modern introduction, Cambridge University Press (1993). MR 95j:53001

[CH] Choi, H. I., Asymptotic Dirichlet problems for harmonic functions on Riemannian manifolds, Trans. Amer. Math. Soc. 281 (1984), 691-716. MR 85b:53040

[EO] P. Eberlain, B. O'Neill, Visibility manifolds, Pacific J. Math 46 (1973), 45-109.

[GW] R.E.Greene, H. Wu, $C^{\infty}$ Approximations of convex, subharmonic and plurisubharmonic functions, Ann. Sci. École Norm. Sup. 12 (1979), 69-100. MR 80m:53055

[HK] E. Heintze, H. Karcher, A general comparison theorem with applications to volume estimates for submanifolds, Ann. Sci. Éc. Norm. Sup., Paris 11 (1978), 451-470. MR 80i:53026

Faculty of Science, Department of Mathematics and Computer Science, Kuwait UniVersity, P.O. Box 5969, Safat 13060, Kuwait

E-mail address: borbely@mcc.sci.kuniv.edu.kw 